\title{
Laser-Induced Graphene-Based Electrochemical Sensor for 4-Nitrophenol from Polyimide and Polyethersulfone Precursors
}

\author{
Vikram P. Wanjari ${ }^{1}$, Ashish Kumar ${ }^{2}$, Siddhartha P. Duttagupta ${ }^{1,3}$, Swatantra P. Singh ${ }^{1.2,4 *}$ \\ ${ }^{1}$ Centre for Research in Nanotechnology and Science, IIT Bombay, Mumbai, India \\ ${ }^{2}$ Environmental Science and Engineering Department, IIT Bombay, Mumbai, India \\ ${ }^{3}$ Department of Electrical Engineering, IIT Bombay, Mumbai, India \\ ${ }^{4}$ Interdisciplinary Program in Climate Studies, IIT Bombay, Mumbai, India
}

*Corresponding Author: Swatantra P. Singh, email: swatantra@iitb.ac.in 


\section{Graphical Abstract:}

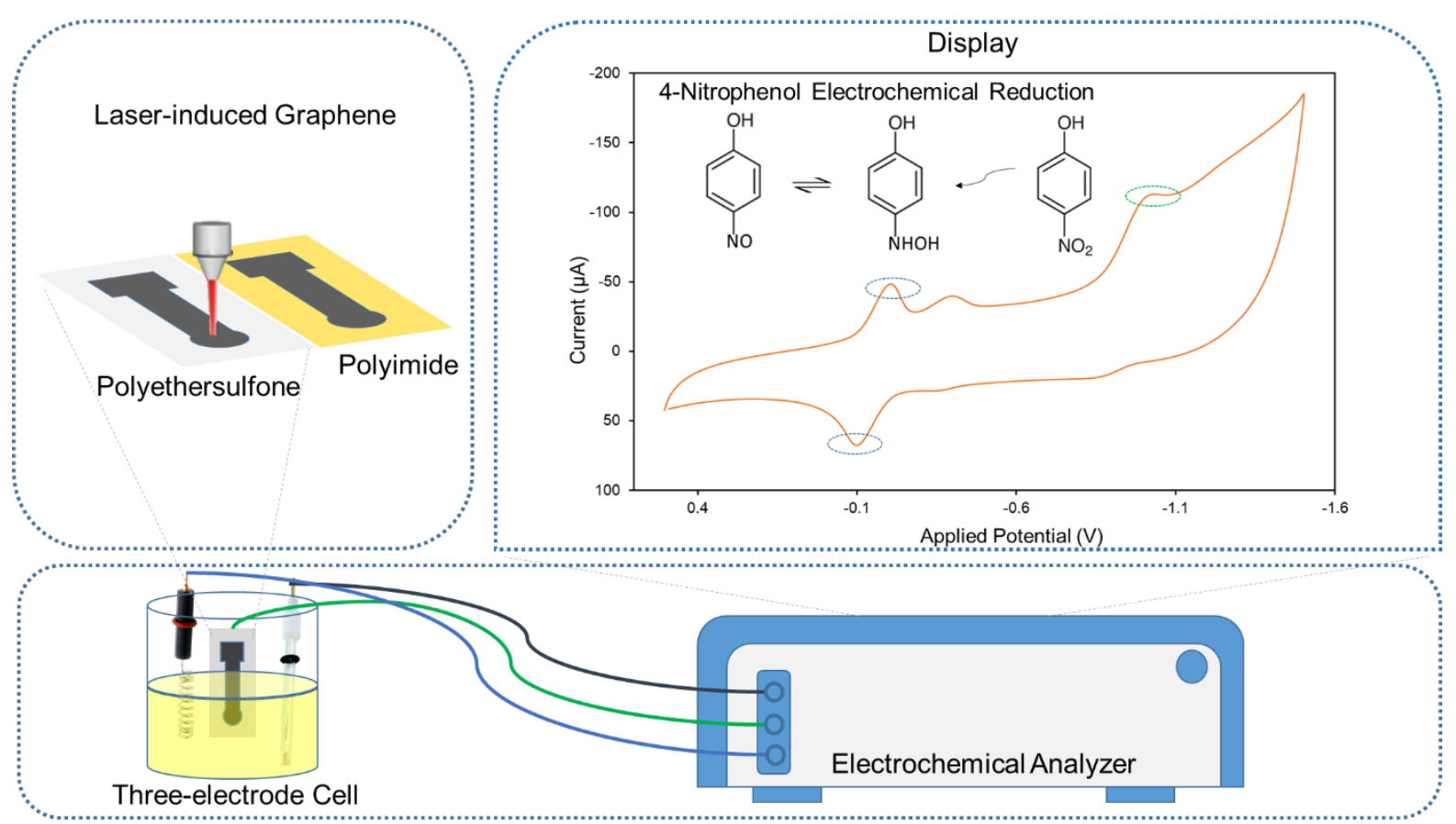




\begin{abstract}
:
Electrochemical sensors provide an excellent alternative for the in-situ detection of pollutants in water. 4-Nitrophenol is a critical pollutant owing to its acute toxicity and adverse health effects on humans and other living organisms. It is known to have carcinogenic, mutagenic, and teratogenic effects on aquatic life plants and human beings at very low concentrations. In this work, a facile electrochemical sensor for 4-Nitrophenol detection is proposed by laser-induced graphene (LIG) printed on polyethersulfone (PES) and polyimide (PI) films respectively. The laser irradiation of polymeric films results in 3D porous graphene structure formation that increases electron transfer rate as well as the electrochemically active surface area (EASA). This fabrication approach by laser-scribing provides a simple, fast, chemical-free, mask-free, and scalable solution to produce graphene-based electrochemical sensors for 4-Nitrophenol. Cyclic voltammetry is used as the electrochemical technique for the highly sensitive detection of 4-Nitrophenol. PES-based LIG sensors exhibit a higher sensitivity of 3793 $\mu \mathrm{AmM}^{-1} \mathrm{~cm}^{-2}$ as compared to that of PI-based LIG sensors with a sensitivity of $3025 \mu \mathrm{AmM}^{-1} \mathrm{~cm}^{-2}$.
\end{abstract}

Keywords: 4-Nitrophenol, Electrochemical sensor, Laser-induced Graphene, Environmental sensor application 


\section{Introduction:}

4-Nitrophenol(4-NP) is a toxic organic compound used extensively as a precursor and intermediate material for the manufacturing of various products, including pesticides, insecticides, dyes, leather, explosives, pharmaceuticals, polymers etc. ${ }^{1,2}$. Hence it is found in the effluents from these industries and introduced into surface waters when insufficiently monitored and untreated. The environmental and health impacts of 4-NP include carcinogenic, mutagenic and teratogenic effects on aquatic life, plant and human beings ${ }^{1}$. The United States Environmental Protection Agency has included 4-NP as one of the priority pollutants ${ }^{3}$.

Many conventional instrumentation methods exist for the detection of 4-NP. These include gas chromatography (GC), high-performance liquid chromatography (HPLC), mass spectrometry, fluorescence, electrophoresis, etc. ${ }^{4}$. However, these methods are time-consuming, require costly instrumentation, complex sample preparation methods, and skilled personnel for operation. The major disadvantage of these techniques lies in the fact that they are not applicable for the on-site detection of pollutants. Electrochemical techniques have received a lot of attention for in-situ detection of pollutants such as phenols, pesticides, heavy metals, etc. ${ }^{5-7}$. The sensors based on electrochemical mechanisms offer the merits of high sensitivity and selectivity, rapid response, simple operation, and low cost.

The detection of 4-Nitrophenol is critical to ensure its removal from the industrial wastewaters and consequent contamination of natural streams. Earlier efforts for electrochemical detection of 4-NP using graphene-based material as electrode have been made in combination with different nanomaterials. Metal nanoparticles such as gold and silver have been used in combination with reduced graphene oxide (rGO). For example, Tang et al. demonstrated a facile method of fabrication of rGO and gold nanocomposite for the electrochemical detection of 4-NP 8 . Jiao et al. performed the electrochemical sensing of 4-NP using rGO-AuNP nanocomposite synthesized by the electrochemical co-reduction method ${ }^{9}$. Ikhsan et al. made use of silver nanoparticles deposited on the rGO surface to develop a 4Nitrophenol electrochemical sensor ${ }^{10}$. Ezhiil-Vilian et al. demonstrated the use of (rGO) modified with palladium nanospheres and gum Arabic (GA) nanocomposite for detection of 4-NP ${ }^{11}$. The use of metal oxide nanoparticles along with graphene is also reported for 4-NP detection. Zaidi et al. used $\mathrm{MnO}_{2}$ 
nanoparticles with graphene, whereas Nehru et al. used a composite of graphene oxide and titanium dioxide $\left(\mathrm{TiO}_{2}\right)$ deposited on a glassy carbon electrode ${ }^{12}$. He et al. fabricated a graphene and acetylene black paste hybrid electrode for 4-NP detection with high sensitivity and selectivity ${ }^{13}$.

Graphene is a 2D nanostructured allotrope of carbon that has excellent electrochemical properties. It also has high electrical conductivity, good chemical stability, and a large surface area. These properties, along with its low cost compared to other materials, make it a viable option for electrochemical sensors. However, conventional fabrication techniques such as mechanical exfoliation, chemical vapor deposition, reduction of graphene oxide, etc., are time-consuming and require chemically intensive steps. Manufacturing large-scale sensors require a simple, fast, and industry scalable option while still offering high sensitivity. Laser-induced graphene (LIG) has garnered increasing interest due to its advantage of simple mask-free and chemical-free fabrication of graphene electrodes ${ }^{14}$. One of the major advantages of LIG lies in the fact that it can be produced from a multitude of substrates ranging from commercial polymers ${ }^{15,16}$ to waste organic materials such as cloth, paper, food,etc ${ }^{17}$. This flexibility offers the potential to explore recyclable and cheaper materials for flexible electronics and other applications $^{18,19}$ Furthermore; porous graphene-based sensors have shown great promise in the development of electrochemical sensors. The porous structure creates efficient multi-dimensional pathways for electron and mass transfer while also creating a higher surface area for the electrochemical interactions between the electrolyte and the electrode surface ${ }^{20}$. The advantage of high electrochemical performance and scalable fabrication process has allowed the detection of many pollutants of environmental significance with improved performance compared to conventional graphene-based sensors $^{21-25}$. To the best of the authors' knowledge, a LIG-based sensor for the detection of 4Nitrophenol hasn't been proposed yet.

This work demonstrated a novel electrochemical sensor for 4-Nitrophenol using LIG as the electrode material. The performances of two different precursor materials for LIG fabrication, viz. polyimide(PI) and polyethersulfone (PES) have been compared. The laser settings were optimized for each of the materials to obtain LIG that gave the best electrochemical performance in the detection of 4Nitrophenol. The sensor demonstrated a high sensitivity of $3025 \mu \mathrm{AmM}^{-1} \mathrm{~cm}^{-2}$ for LIG-PI and 3793 
$\mu \mathrm{AmM}^{-1} \mathrm{~cm}^{-2}$ for LIG-PES sensor with a limit of detection of $0.1 \mathrm{mM}$ using cyclic voltammetry technique.

\section{Materials and methods}

\section{Materials:}

Polyimide (PI) sheets of $127 \mu \mathrm{m}$ thickness were purchased from Dali Electronics, India. Polyethersulfone (PES), Dichloromethane, 4-Nitrophenol (4-NP), Potassium ferricyanide $\left(\mathrm{K}_{3} \mathrm{Fe}(\mathrm{CN})_{6}\right)$, Potassium Chloride $(\mathrm{KCl})$ were purchased from LOBA Chemie, India. All chemicals used were of AR/ACS grade.

\section{PES sheet fabrication:}

PES Sheets were fabricated by dissolving $2.65 \mathrm{gm}$ PES powder in a $22 \mathrm{ml}$ dichloromethane solution. The resulting mixture was poured into a glass Petri plate and allowed to consolidate by limited exposure to the ambient atmosphere. PES sheets as prepared and polyimide sheets as obtained from the manufacturer were used for laser scribing after cleaning the surface with ethanol.

\section{LIG fabrication using PES and PI sheets:}

The substrate PES and PI were scribed with $10.6 \mu \mathrm{m} \mathrm{CO}_{2}$ laser with a VLS 3.50 laser platform equipped with 50 W, 2.0 inch Lens kit (Universal Laser Systems, (Distributor: Caliber, Tel Aviv, Israel)). The laser settings were adjusted to optimize the conductivity of LIG and the stability of the electrodes during electrochemical operation. The LIG electrodes were designed to have a working surface area of $3 \mathrm{~mm}$ diameter and a connecting length of $10 \mathrm{~mm}$. The connecting area of the LIG was passivated using insulating adhesive tape. The material characterization of fabricated LIG electrodes was done by scanning electron microscopy (SEM). Electrochemical characterization of the electrodes was performed using cyclic voltammetry.

Optimal laser settings are required to produce the highest quality of LIG on either of the polymer substrates, viz. PES and PI. In order to get the best output of LIG fabrication, the power settings and scan speeds were adjusted to different levels, and the resistance of the resulting electrode was measured. 
Square dimensions of $1 \mathrm{~cm} \times 1 \mathrm{~cm}$ were laser scribed onto the PES sheet using $20 \%$ power and $25 \%$ scan speed. The settings were tried with a $1 \%$ step increase in power and a subsequent decrease in speed of the same magnitude. The increase in power presumably leads to more penetration of the polymer thickness, and hence more amount of graphene is produced. A similar effect is possible due to reduced speed as the energy of a slower-moving laser can accumulate and penetrate deeper into the polymer depth. The optimal settings for LIG formation on the PES sheet were found to be at $20 \%$ power and 25 $\%$ speed. The procedure of optimization of LIG formation was similarly performed on the PI sheet. Settings at which optimal LIG formation was observed on PI were $17 \%$ power and $25 \%$ speed.

Increasing the power of the laser is known to cause more penetration into the polymer sheet, thus generating more amount of graphene. However, if the power is exceedingly high, the graphene formation is unstable and collapses easily with minor deformation. Further, increase in power results in complete burning off the substrate, causing complete dismantling of the electrode structure ${ }^{20}$.

\section{Electrochemical setup:}

Cyclic voltammetry analysis was carried out using a Multi Autolab 204 electrochemical analyzer (Metroohm, India). A three-electrode setup was used for the electrochemical characterization as well as the sensor performance studies. Electrochemical measurements were taken at room temperature. LIG was used as the working electrode, saturated calomel electrode (SCE) was used as the reference electrode, and platinum coil as the counter electrode. The electrochemical cell had an electrolyte solution of $80 \mathrm{ml}$ volume. Activation of LIG electrodes was carried out prior to performing the electrochemical procedures. Cyclic voltammetry runs at $100 \mathrm{mV} / \mathrm{s}$ were performed for 30 cycles to activate the LIG electrodes and remove any organic binder impurities. 


\section{Results and Discussion:}

\section{Characterization}

\section{Material Characterization:}

SEM images of the surface modifications show the formation of porous structure on polyethersulfone surface (Fig. 1a and 1b). This observed structure is in line with previously reported LIG-based work using PES ${ }^{26,27}$. The porous nanofibre-like structure allows for increased interaction of the electrolyte with the electrode surface. In the SEM image, the porous structure on the surface with abundant edgeplane sites is visible. The focused laser beam acting on the polymer surface produces a temperature at more than $2500^{\circ} \mathrm{C}$, resulting in burning off some of the carbon in the presence of ambient oxygen and moisture. The burning produces $\mathrm{CO}_{2}$ and other gases, which results in the porous nature of the graphene surface while still maintaining considerable structural stability depending on the optimization of laser parameters2. A stable 3D morphological structure facilitates a high electron transfer rate, and the porous nature increases the electrochemically active surface area.
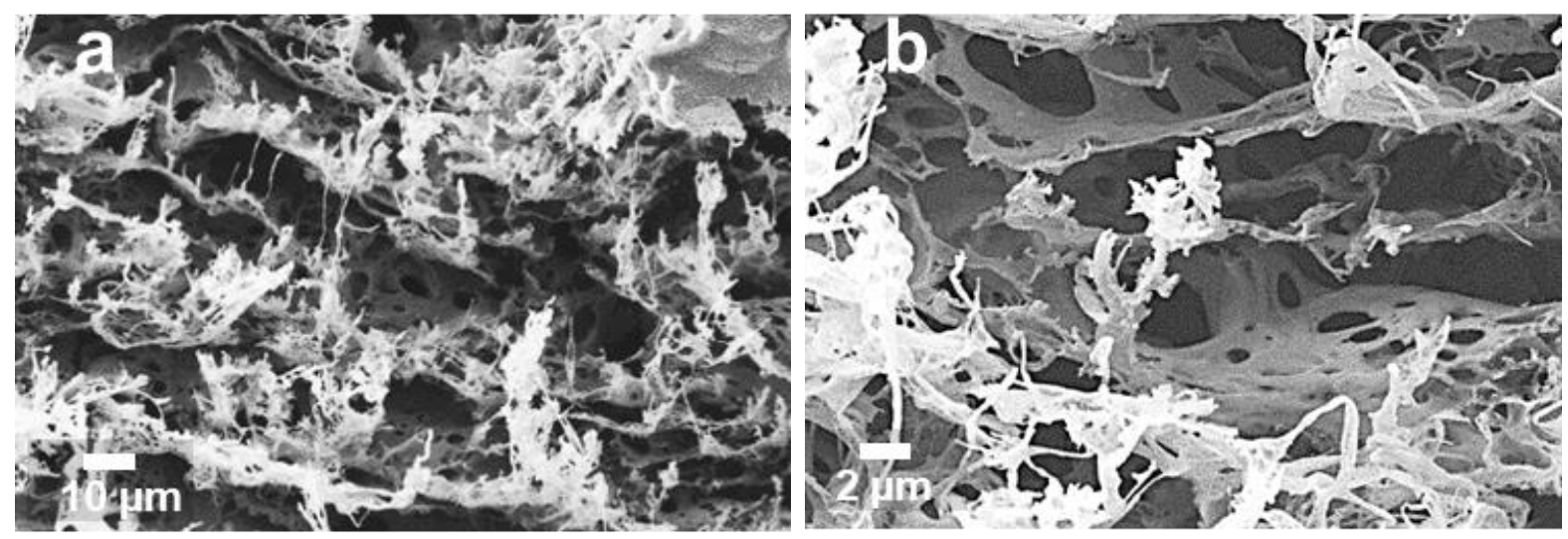

Figure 1. (a,b) SEM top-view images at different resolutions for laser-induced graphene fabricated on PES substrate 


\section{Electrochemical characterization:}

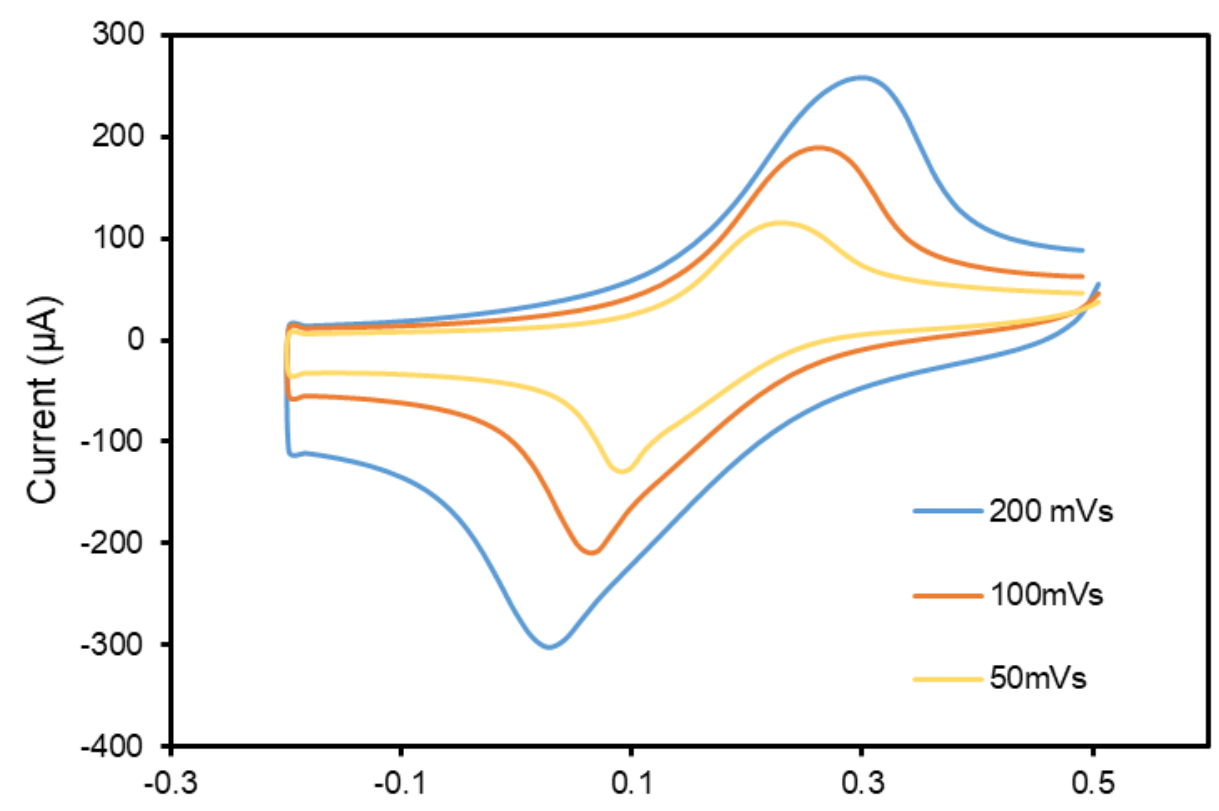

Figure 2. Cyclic voltammetry responses using LIG-PES for $5 \mathrm{mM} \mathrm{K}_{3} \mathrm{Fe}(\mathrm{CN})_{6}$ at different scan rates

The electrochemical characterization of the fabricated LIG electrodes is typically carried out using a model redox probe of potassium ferricyanide in potassium chloride ${ }^{29}$. Figure 2 shows the electrochemical characterization of LIG-PES by cyclic voltammetry. $5 \mathrm{mM}$ potassium ferricyanide $\mathrm{K}_{3} \mathrm{Fe}(\mathrm{CN})_{6}$ was used along with $0.1 \mathrm{M} \mathrm{KCl}$ to perform the experiment. The increasing scan rate results in the shifting of the oxidation peak to become more positive, whereas the reduction peak shifts to a more negative side as expected ${ }^{30}$. The electrocatalytic properties of LIG were evaluated using the cyclic voltammetry technique. Fig 3 shows the generation of cyclic voltammetry signals in $1 \mathrm{mM} 4-\mathrm{NP}$ solution using LIG-PES electrodes at a scan rate of $100 \mathrm{mV} / \mathrm{s}$. An irreversible discernible peak was obtained at around $-1.0 \mathrm{~V}$, whereas reversible cathodic and anodic peaks were obtained around $-0.2 \mathrm{~V}$ and $-0.1 \mathrm{~V}$, respectively. The presence of these two types of characteristic peaks conforms to the findings in the literature where graphene-based electrodes generate similar peaks for 4-Nitrophenol ${ }^{12}$. 


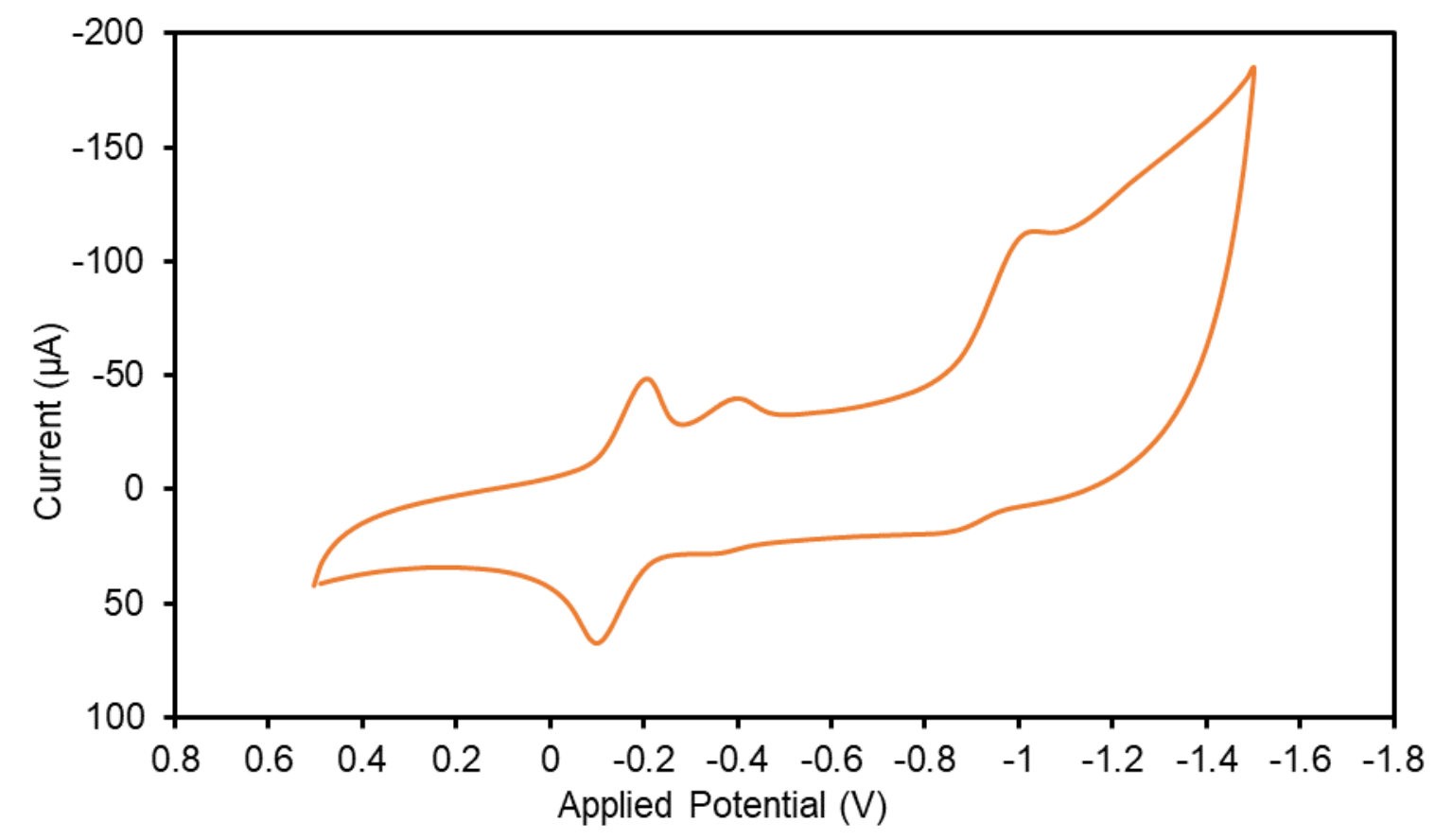

Figure 3. Cyclic voltammetry responses using LIG-PES for $1 \mathrm{mM} 4-\mathrm{NP}$ at $100 \mathrm{mV} / \mathrm{s}$

\section{Sensor performance by cyclic voltammetry:}

4-Nitrophenol was dissolved in $0.1 \mathrm{M} \mathrm{KCl}$ solution at different concentrations from $1 \mathrm{mM}$ to $0.1 \mathrm{mM}$. Cyclic voltammetry was performed using LIG as working electrode, Saturated calomel electrode (SCE) as reference electrode, and platinum coil as the counter electrode. The voltage was varied from $-1.2 \mathrm{~V}$ to $+0.5 \mathrm{~V}$ at a scan rate of $10 \mathrm{mV} / \mathrm{s}$ during the measurements.

Cyclic voltammetry (CV) peak measurements at different concentrations of 4-Nitrophenol are shown in Figures $4 \mathrm{a}$ and $4 \mathrm{~b}$ for PES and PI-based LIG electrodes, respectively. The CV peaks in the case of LIG-PI are found to be visually more prominent as compared to the peaks obtained using LIG-PES. However, from the response current to input concentration ratio, the sensitivity of LIG-PES at $0.1 \mathrm{mM}$ concentration of 4-NP is $3793 \mu \mathrm{AmM}^{-1} \mathrm{~cm}^{-2}$, which is significantly higher than $3025 \mu \mathrm{AmM}^{-1} \mathrm{~cm}^{-2}$ for LIG-PI. The higher sensitivity of LIG-PES toward 4-NP may be due to the presence of sulfur groups on the graphene surface of LIG-PES. From the graph, the sensor's limit of detection (LoD) is found to be $100 \mu \mathrm{M}$. The PES-based LIG electrode sensor demonstrated a slightly wider linear range up to $1 \mathrm{mM}$ as against the PI-based LIG electrode with up to $0.9 \mathrm{mM}$ upper range. The LoD was estimated based on 
the minimum detectable response of the sensor, which is three times the RMS deviation in the baseline response.

The calibration curves have been shown in the insets of Figures $4 \mathrm{a}$ and $4 \mathrm{~b}$. These analytical curves can be obtained by plotting the peak currents for different concentrations of 4-NP under optimized experimental conditions ${ }^{31}$. The cathodic peak current is proportional to the concentration in the range of 0.1 - $1 \mathrm{mM}$ for LIG-PES and $0.1-0.9 \mathrm{mM}$ for LIG-PI. The linear regression equation for LIG-PES can be expressed as $\mathrm{I}_{\mathrm{PC}}(\mu \mathrm{A})=-21.249 \mathrm{C}(\mathrm{mM})-24.991$, with a correlation coefficient $\left(\mathrm{R}^{2}\right)$ of 0.9977 . The linear regression equation for LIG-PI can be expressed as $\operatorname{IPC}_{\mathrm{PC}}(\mu \mathrm{A})=-32.101 \mathrm{C}(\mathrm{mM})-17.736$, with a correlation coefficient $\left(\mathrm{R}^{2}\right)$ of 0.9962 . The efficient performance of the proposed LIG-based sensors can be attributed to the electrocatalytic properties and adsorption ability of graphene in porous form. LIG possesses a high electrochemical response due to its porous structure, which offers more sites for electrochemical redox reactions as compared to conventional planar graphene ${ }^{32}$. The porous structure also offers more edge defects than basal defects which can be related to the enhanced electrochemical activity. 

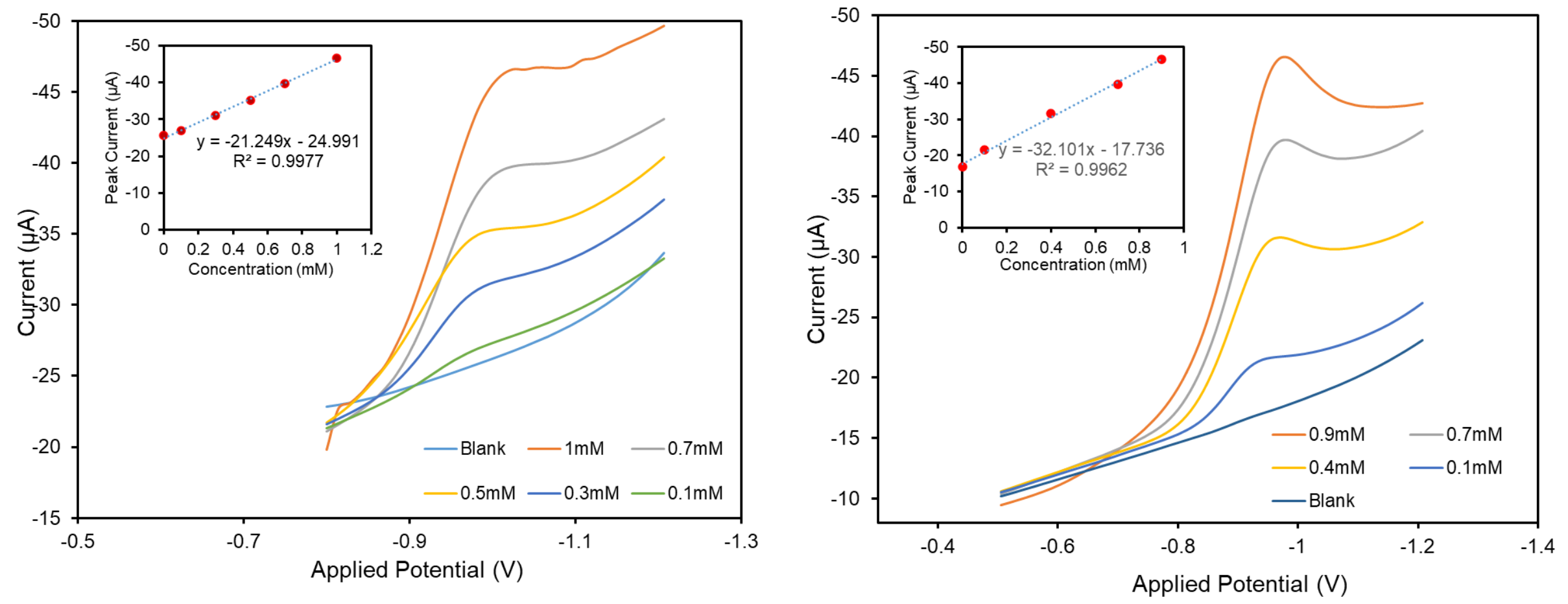

Figure 4. Cyclic voltammetry responses using a) LIG-PES and b) LIG-PI for different concentrations of 4-nitrophenol 


\section{Conclusion:}

In summary, a flexible miniaturized electrochemical sensor for the detection of 4-Nitrophenol is proposed using LIG from two polymeric substrates viz. PES and PI. The fabrication process is simple, one-step, scalable, chemical-free as well as mask-free. The laser-scribing process creates 3D morphological graphene, which facilitates a higher electron transfer rate and increased electrochemically active surface area (EASA). The PES-based LIG sensor shows higher sensitivity than PI-based LIG in the detection of 4-NP. The limit of detection achieved by the sensors is $100 \mu \mathrm{M}$. Since the electrochemical cell used in this work consists of standard reference and counter electrodes, future work shall develop a 3-electrode system derived from LIG and evaluate its performance. The sensor has the potential to evolve into a dual or multi-pollutant detection sensor by identifying characteristic redox peaks of different environmental pollutants. 


\section{References:}

1. Ramalingam, M., Ponnusamy, V. K. \& Sangilimuthu, S. N. Electrochemical determination of 4-nitrophenol in environmental water samples using porous graphitic carbon nitride-coated screen-printed electrode. Environmental Science and Pollution Research 27, 17481-17491 (2020).

2. Manjula, N. \& Chen, S. M. Simple strategy synthesis of manganese cobalt oxide anchored on graphene oxide composite as an efficient electrocatalyst for hazardous 4-nitrophenol detection in toxic tannery waste. Microchemical Journal 168, (2021).

3. Dong, Z. et al. Ni@Pd core-shell nanoparticles modified fibrous silica nanospheres as highly efficient and recoverable catalyst for reduction of 4-nitrophenol and hydrodechlorination of 4chlorophenol. Applied Catalysis B: Environmental 162, 372-380 (2015).

4. Devadas, B., Rajkumar, M., Chen, S. M. \& Yeh, P. C. A novel voltammetric p-nitrophenol sensor based on $\mathrm{ZrO} 2$ nanoparticles incorporated into a multiwalled carbon nanotube modified glassy carbon electrode. Analytical Methods 6, 4686-4691 (2014).

5. Mahmoudi, E., Fakhri, H., Hajian, A., Afkhami, A. \& Bagheri, H. High-performance electrochemical enzyme sensor for organophosphate pesticide detection using modified metalorganic framework sensing platforms. Bioelectrochemistry 130, (2019).

6. Ensafi, A. A., Akbarian, F., Heydari-Soureshjani, E. \& Rezaei, B. A novel aptasensor based on 3D-reduced graphene oxide modified gold nanoparticles for determination of arsenite. Biosensors and Bioelectronics 122, 25-31 (2018).

7. Chen, K., Zhang, Z. L., Liang, Y. M. \& Liu, W. A graphene-based electrochemical sensor for rapid determination of phenols in water. Sensors (Switzerland) 13, 6204-6216 (2013).

8. Tang, Y. et al. Electrochemical detection of 4-nitrophenol based on a glassy carbon electrode modified with a reduced graphene oxide/Au nanoparticle composite. Analytical Methods $\mathbf{5}$, 5508-5514 (2013).

9. Jiao, X. X., Luo, H. Q. \& Li, N. B. Fabrication of graphene-gold nanocomposites by electrochemical co-reduction and their electrocatalytic activity toward 4-nitrophenol oxidation. Journal of Electroanalytical Chemistry 691, 83-89 (2013).

10. Ikhsan, N. I., Rameshkumar, P. \& Huang, N. M. Controlled synthesis of reduced graphene oxide supported silver nanoparticles for selective and sensitive electrochemical detection of 4nitrophenol. Electrochimica Acta 192, 392-399 (2016).

11. Vilian, A. T. E. et al. Pd nanospheres decorated reduced graphene oxide with multi-functions: Highly efficient catalytic reduction and ultrasensitive sensing of hazardous 4-nitrophenol pollutant. Journal of Hazardous Materials 333, 54-62 (2017).

12. Nehru, R., Gopi, P. K. \& Chen, S. M. Enhanced sensing of hazardous 4-nitrophenol by a graphene oxide-TiO2 composite: Environmental pollutant monitoring applications. New Journal of Chemistry 44, 4590-4603 (2020).

13. He, Q. et al. Facile and ultrasensitive determination of 4-nitrophenol based on acetylene black paste and graphene hybrid electrode. Nanomaterials 9, (2019).

14. Lin, J. et al. Laser-induced porous graphene films from commercial polymers. Nature Communications 5, (2014). 
15. Singh, S. P. et al. Laser-Induced Graphene Layers and Electrodes Prevents Microbial Fouling and Exerts Antimicrobial Action. ACS Applied Materials and Interfaces 9, 18238-18247 (2017).

16. Rathinam, K. et al. Polyimide derived laser-induced graphene as adsorbent for cationic and anionic dyes. Carbon 124, 515-524 (2017).

17. Chyan, Y. et al. Laser-Induced Graphene by Multiple Lasing: Toward Electronics on Cloth, Paper, and Food. ACS Nano 12, 2176-2183 (2018).

18. Dixit, N. \& Singh, S. P. Laser-Induced Graphene (LIG) as a Smart and Sustainable Material to Restrain Pandemics and Endemics: A Perspective. ACS Omega acsomega.1c06093 (2022) doi:10.1021/acsomega.1c06093.

19. Barbhuiya, N. H. et al. Virus inactivation in water using laser-induced graphene filters. Materials 14, (2021).

20. Lu, Z. et al. Ionic liquid/poly-L-cysteine composite deposited on flexible and hierarchical porous laser-engraved graphene electrode for high-performance electrochemical analysis of lead ion. Electrochimica Acta 295, 514-523 (2019).

21. Nasraoui, S. et al. Electrochemical sensor for nitrite detection in water samples using flexible laser-induced graphene electrodes functionalized by CNT decorated by Au nanoparticles. Journal of Electroanalytical Chemistry 880, (2021).

22. Ge, L., Hong, Q., Li, H., Liu, C. \& Li, F. Direct-Laser-Writing of Metal Sulfide-Graphene Nanocomposite Photoelectrode toward Sensitive Photoelectrochemical Sensing. Advanced Functional Materials 29, (2019).

23. Cheng, C. et al. Bisphenol A Sensors on Polyimide Fabricated by Laser Direct Writing for Onsite River Water Monitoring at Attomolar Concentration. ACS Applied Materials and Interfaces 8, 17784-17792 (2016).

24. Lu, Z. et al. Ionic liquid/poly-L-cysteine composite deposited on flexible and hierarchical porous laser-engraved graphene electrode for high-performance electrochemical analysis of lead ion. Electrochimica Acta 295, 514-523 (2019).

25. Cardoso, A. R. et al. Molecularly-imprinted chloramphenicol sensor with laser-induced graphene electrodes. Biosensors and Bioelectronics 124-125, 167-175 (2019).

26. Singh, S. P., Li, Y., Zhang, J., Tour, J. M. \& Arnusch, C. J. Sulfur-Doped Laser-Induced Porous Graphene Derived from Polysulfone-Class Polymers and Membranes. ACS Nano 12, 289-297 (2018).

27. Grossman, J. C., Getachew, B. A. \& Bergsman, D. S. Laser-induced graphene from polyimide and polyethersulfone precursors as a sensing electrode in anodic stripping voltammetry. ACS Applied Materials and Interfaces 12, 48511-48517 (2020).

28. Nayak, P., Kurra, N., Xia, C. \& Alshareef, H. N. Highly Efficient Laser Scribed Graphene Electrodes for On-Chip Electrochemical Sensing Applications. Advanced Electronic Materials 2, (2016).

29. Nasraoui, S. et al. Electrochemical sensor for nitrite detection in water samples using flexible laser-induced graphene electrodes functionalized by CNT decorated by Au nanoparticles. Journal of Electroanalytical Chemistry 880, (2021). 
30. Soares, R. R. A. et al. Laser-Induced Graphene Electrochemical Immunosensors for Rapid and Label-Free Monitoring of Salmonella enterica in Chicken Broth. ACS Sensors 5, 1900-1911 (2020).

31. Li, J. et al. A graphene oxide-based electrochemical sensor for sensitive determination of 4nitrophenol. Journal of Hazardous Materials 201-202, 250-259 (2012).

32. Zhu, X. et al. Portable wireless intelligent sensing of ultra-trace phytoregulator $\alpha$-naphthalene acetic acid using self-assembled phosphorene/Ti3C2-MXene nanohybrid with high ambient stability on laser induced porous graphene as nanozyme flexible electrode. Biosensors and Bioelectronics 179, (2021). 